Gynecology

\title{
Epidemiology and management of uterine fibroids
}

\author{
Emma Giuliani $^{1}$ | Sawsan As-Sanie ${ }^{2}$ | Erica E. Marsh ${ }^{1, *}$
}

${ }^{1}$ Division of Reproductive Endocrinology and Infertility, Department of Obstetrics and Gynecology, University of Michigan, Ann Arbor, MI, USA

${ }^{2}$ Division of Gynecology, Department of Obstetrics and Gynecology, University of Michigan, Ann Arbor, MI, USA

\section{${ }^{*}$ Correspondence}

Erica E. Marsh, Division of Reproductive Endocrinology and Infertility, Department of Obstetrics and Gynecology, University of Michigan, Ann Arbor, MI, USA.

Email:marshee@med.umich.edu

\begin{abstract}
Uterine leiomyomas are one of the most common and yet understudied diseases in women. These tumors, commonly known as fibroids, affect women mainly during their reproductive years and are diagnosed in up to $70 \%$ of white women and more than $80 \%$ of women of African ancestry during their lifetime. This disease has a profound impact on health care delivery and costs worldwide. Though most women with fibroids are asymptomatic, approximately $30 \%$ of them will present with severe symptoms which can include abnormal uterine bleeding, anemia, pelvic pain and pressure, back pain, urinary frequency, constipation, or infertility, and will require intervention. Furthermore, fibroids have been associated with poor obstetrical outcomes. The current options for symptomatic fibroid treatment include expectant, medical, and surgical management, and interventional radiology procedures. This article reviews the recent progress and available management strategies for uterine fibroids and highlights areas where further research is needed to find new therapeutic targets and better personalize treatments.
\end{abstract}

KEYWORDS

Abnormal uterine bleeding; Leiomyomas; Uterine fibroids

\section{1 | INTRODUCTION}

Leiomyomas, more commonly known as fibroids, are the most prevalent solid gynecological benign tumors of the uterus. They originate from clonal expansion of a single cell in the myometrium. ${ }^{1,2}$ Because of their hormonally-responsive nature, fibroids mainly affect women during their reproductive years, are extremely rare prior to menarche, and typically regress following menopause. ${ }^{3}$

\section{1 | Epidemiology}

The prevalence of fibroids has been historically underestimated by epidemiologic studies which focused mainly on symptomatic women, leaving behind a large population of asymptomatic women and women who underreport their symptoms. ${ }^{1,4}$ The prevalence of fibroids varies among different studies and countries (4.5\%-68.6\%) based on the type of investigation, method of diagnosis, and racial/ ethnic demographics of the population studied. ${ }^{5}$ Recent research from the United States has shown that fibroids are detected by ultrasound in more than $80 \%$ of women of African ancestry and nearly $70 \%$ of white women by the age of $50 .{ }^{6}$ Limited self-reported data are available on the prevalence of fibroids in other racial/ethnic groups such as Asian and Hispanic women. This is clearly an area of opportunity for future research and collaborations. The high prevalence of fibroids has a profound impact on health care costs worldwide. Studies have estimated annual direct and indirect costs related to uterine fibroids to be as high as US $\$ 34.4$ billion in the United States, and total annual costs related to hospital admissions of US \$348 million in Germany, US \$120 million in France, and US \$86 million in England. ${ }^{7,8}$ Further cost analysis studies are needed to better describe the economic burden of this condition in other geographic areas such as Africa, Asia, and Latin America.

There are notable racial differences in the prevalence and presentation of fibroids. ${ }^{1}$ Fibroids are more common, tend to present at a younger age, are greater in number, and larger in size in women of African ancestry versus white or Asian women. ${ }^{6,9}$ Other risk factors include obesity, nulliparity, hypertension, late menopause, early menarche, family history of fibroids, and older age. ${ }^{1}$ The impact of diet, 
exercise, smoking, alcohol, stress, and other environmental factors on the pathogenesis of uterine fibroids remains less clear. ${ }^{10}$

Despite their prevalence, relatively little is known about the specific factors and molecular mechanisms that regulate fibroid development, growth, and regression. ${ }^{11}$ Multiple in vitro and in vivo studies have identified several pathways and mechanisms thought to be involved in the pathogenesis of fibroids including sex hormones, stem cells, glucocorticoids, growth factors, cytokine signaling, extracellular matrix remodeling, and epigenetic factors. ${ }^{1,2,11,12}$ Another peculiar characteristic of fibroids is their biological heterogeneity even among those within the same woman's uterus, as shown by different patterns of growth over time. ${ }^{13}$ These considerations highlight the need for more personalized and fibroid-specific treatments.

\section{2 | Symptoms and patients' experience}

Up to $70 \%$ of fibroids are asymptomatic and may be incidentally diagnosed during radiologic procedures conducted for other indications. ${ }^{1,14}$ Asymptomatic fibroids do not require treatment or frequent follow-up. Symptomatic fibroids, based on their size and location in the uterus, can manifest with bulk symptoms (pelvic pressure, back or abdominal pain, fullness), abnormal uterine bleeding (AUB), dysmenorrhea, bladder or bowel symptoms (urinary frequency or constipation), and sexual dysfunction, or can be associated with infertility and other poor obstetrical outcomes such as increased risk of preterm labor, cesarean delivery, antepartum bleeding, fetal malpresentation, and growth restriction. ${ }^{14}$ Several studies have shown that women with fibroids, as a result of their associated symptomatology, have a higher risk of developing emotional distress, depression, and anxiety, which can strongly impact their quality of life. ${ }^{15}$

\section{3 | Evaluation and classification}

Careful collection of medical history/symptoms, pelvic examination, ultrasound, and/or saline-infusion sonogram are very important when planning a targeted treatment for fibroids. ${ }^{14}$ While ultrasound is the most commonly used first line radiologic evaluation for uterine fibroids and in most cases sufficient for their diagnosis (90\%-99\% sensitivity), it is limited in its ability to assess fibroid viability/blood supply which would be necessary for counseling a patient on the likelihood of success of uterine artery embolization (UAE) and/or magnetic resonance guided focused ultrasound radiofrequency ablation (MRgFUS). ${ }^{14}$ Saline-infusion sonogram is a very sensitive and specific procedure for the detection of submucosal fibroids (98\%-100\%). ${ }^{14}$ Magnetic resonance imaging (MRI), while significantly more expensive and cost-prohibitive in certain geographic areas, is more precise for fibroid mapping, fibroid viability assessment, and identification of co-existing uterine pathology such as adenomyosis which are important factors to evaluate in women who are considering conservative surgical management. ${ }^{14}$ Although numerous fibroid classifications can be found in the literature, the most recent and most widely adopted is the 2011 International Federation of Gynecology and Obstetrics (FIGO) classification which describes eight classes of fibroids while accounting for their location and degree of intramural/intracavitary extension (Fig. 1). ${ }^{16}$

\section{I MANAGEMENT}

The treatment of fibroids should not only be directed toward improving symptomatology, but also influenced by the patient's desire for future fertility, desire to retain the uterus, likelihood of achieving treatment goals, and overall health status. Treatment options improve fibroid-associated symptomatology by reducing the size of the fibroids, controlling fibroid-related AUB, or definitively curing the fibroids. Available treatments include medical therapies, interventional radiology, and surgical procedures (Fig. 2). A step-up approach is recommended by many international obstetrical and gynecology societies when treating uterine fibroids, ${ }^{17}$ which begins with pharmacological and minimally invasive treatments before moving to surgery. Despite the advancement in medical and uterine-preserving treatment options in the past decade, hysterectomy remains the most commonly offered and chosen treatment by women with symptomatic fibroids. ${ }^{17}$ Approximately one-third of all hysterectomies worldwide are performed for an underlying diagnosis of uterine fibroids. ${ }^{18}$

\section{1 | Medical management of uterine fibroids and their symptoms}

Medical management primarily aims to reduce the AUB caused by fibroids. However, there are extremely limited studies that have specifically investigated fibroid-related AUB in response to medical treatment as most data on AUB certainly include women with fibroids but also women with $A \cup B$ as a result of other causes. Available medical treatments include anti-fibrinolytic agents, nonsteroidal anti-inflammatory drugs (NSAIDs), combined hormonal

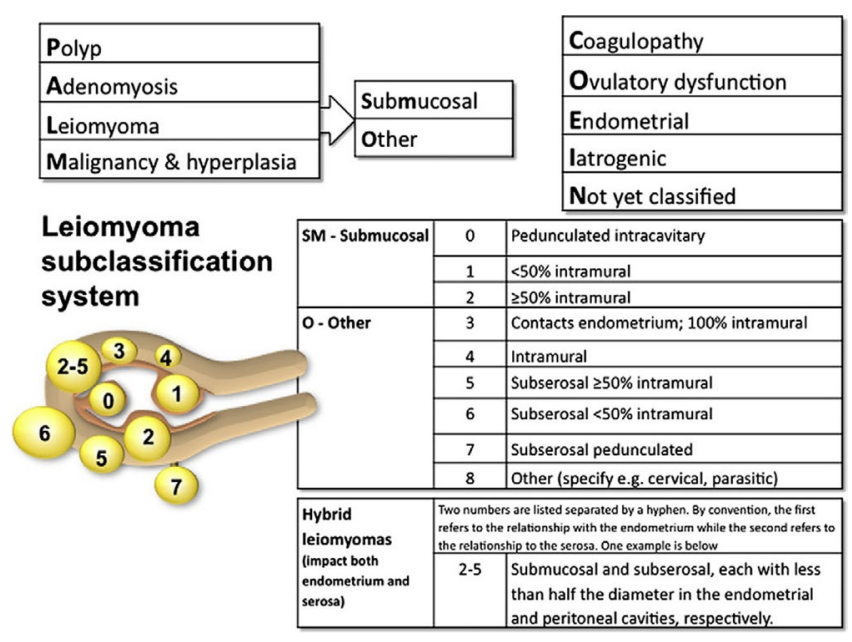

FIGURE 1 International Federation of Gynecology and Obstetrics (FIGO) classification system (PALM-COEIN) for causes of abnormal uterine bleeding including leiomyoma subclassification system. ${ }^{16}$ Copyright permission was obtained to reproduce this figure. 

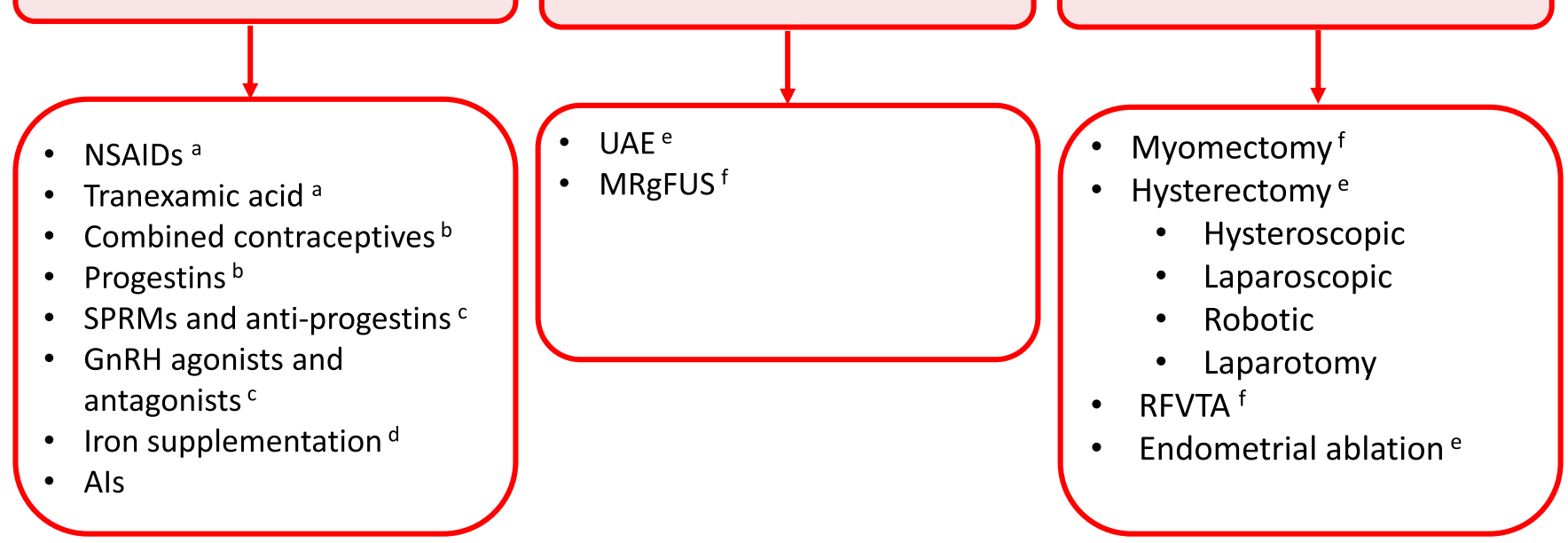

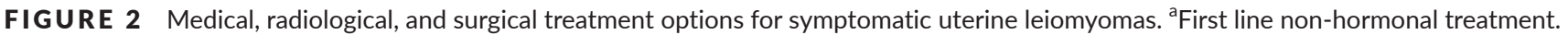

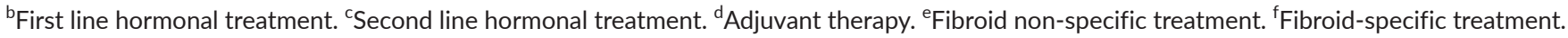
Abbreviations: Als, aromatase inhibitors; GnRH, gonadotropin releasing hormone; MRgFUS, magnetic resonance guided focused ultrasound radiofrequency ablation; NSAIDs, non-steroidal anti-inflammatory drugs; RFVTA, radiofrequency volumetric thermal ablation; SPRMs, selective progesterone receptor modulators; UAE, uterine artery embolization.

contraceptives, progesterone-only treatments, selective progesterone receptor modulators (SPRMs), anti-progestins, aromatase inhibitors, and gonadotropin releasing hormone $(\mathrm{GnRH})$ agonists or antagonists.

In addition to bleeding control, fibroid shrinkage can be seen with $\mathrm{GnRH}$ agonists/antagonists, SPRMs, and aromatase inhibitors. ${ }^{18}$ Other pharmaceutical agents that have been used for the treatment of fibroids fall into the categories of androgens, estrogen receptor antagonists, and selective estrogen receptor modulators. However, these classes of medications offer limited advantages for improving heavy bleeding over their better studied counterparts and their use is limited as a result of potential adverse effects. Novel medical targets, currently under investigation, include green tea extract, vitamin D, cabergoline, gestrinone, and somatostatin analogs. ${ }^{19}$ While we are presenting data on most medical therapies available, we note that not all therapies are approved in all countries and that significant variations in cost impact access and utilization.

\subsection{1 | Non-steroidal anti-inflammatory agents}

First line medical management for AUB and dysmenorrhea induced by fibroids involves the use of NSAIDs due to their low cost, limited adverse effects, and general availability. ${ }^{19}$ The most commonly used agents are ibuprofen (600-1800 mg daily) or naproxen (550-1100 mg daily) which are most efficacious when started a day or two before the onset of menses and continued for the duration of menstruation. ${ }^{20}$ NSAIDs work by inhibiting the enzyme cyclooxygenase and lowering the production of pro-inflammatory prostaglandins and have been shown to improve dysmenorrhea and menorrhagia compared to placebo. ${ }^{20}$ However, they are less effective in reducing menstrual bleeding than tranexamic acid, combined hormonal contraceptives, or levonorgestrel intrauterine devices. ${ }^{20}$ NSAIDs should be avoided in women with known hypersensitivity to this class of medications, active gastric or peptic ulcers, or renal disease.

\subsection{2 | Tranexamic acid}

The synthetic lysine derivative, tranexamic acid, is an antifibrinolytic agent and promoter of blood clot formation. ${ }^{19}$ It is one of the oldest and most globally available treatments of AUB. By preventing fibrin degradation at the level of the plasminogen lysine receptor site, it favors pro-coagulant mechanisms which lead to a reduction in menstrual blood flow and improvement of symptoms. ${ }^{21}$ Most commonly, it is administered at a dose of two $650 \mathrm{mg}$ tablets orally three times a day for up to 5 days. ${ }^{21}$ It is associated with rare and mild adverse effects including gastrointestinal and musculoskeletal symptoms, and it is contraindicated for patients with color blindness, active bleeding, history of intravascular clotting, or hypersensitivity to the medication. ${ }^{21}$

\subsection{3 | Combined hormonal contraceptives}

Combined estrogen-progesterone contraceptives, in the form of pills, vaginal devices, or transdermal patches, used cyclically or continuously, are commonly used for the treatment of AUB, including in women with uterine fibroids. ${ }^{13}$ They mainly tend to keep the endometrium thin and decrease the amount of endometrial shedding during the menstrual cycle. Fibroid-associated AUB, hemoglobin concentration, and quality of life were found to be improved in women who used combined oral contraceptives compared to placebo, however they performed less well than progestin-releasing intrauterine devices (IUD). ${ }^{22}$ Medical eligibility criteria such as age, smoking, history of 
venous thrombosis, and migraines with aura should be reviewed with the patient before recommending the use of any combined hormonal contraceptives as well as adverse effects including nausea, headaches, and irregular bleeding. ${ }^{22}$

\subsection{4 | Progestins}

The most common progesterone-only treatments for AUB involve the use of oral progestins (norethindrone acetate 5 to $10 \mathrm{mg}$ daily; medroxyprogesterone acetate $10 \mathrm{mg}$ daily; megestrol $40 \mathrm{mg}$ daily) and progesterone-releasing IUDs. ${ }^{23}$ Data on progestin-only injectable medications showed high discontinuation rate due to irregular bleeding profile making them a less ideal option. ${ }^{17}$ By repressing the estrogenic stimulated growth of the endometrium, this class of medications consequently decreases blood loss during menstruation. Some of the most common adverse effects of this class of medications are gastrointestinal symptoms, irregular bleeding, acne, breast tenderness, and mood changes. ${ }^{17}$ Levonorgestrel-releasing IUDs, by acting locally on the endometrium, with minimal systemic absorption and adverse effects, cause amenorrhea and/or improvement of menorrhagia and anemia in up to $50 \%-60 \%$ of patients with AUB at 6-12 months, including women with fibroids. ${ }^{23}$ Unfortunately, several studies reported higher expulsion rates in women with fibroids, especially with fibroids larger than $3 \mathrm{~cm}$, compared to women without fibroids $(6 \%-12 \% \text { vs } 0 \%-3 \%)^{23}$

\subsection{5 | Selective progesterone receptor modulators and anti-progestins}

SPRMs and anti-progestins act at the level of the peripheral progesterone receptors by inducing apoptosis, inhibiting cellular proliferation of the fibroid and thinning the endometrial lining. ${ }^{24}$ Ulipristal acetate (5-10 mg orally once a day) has been associated with $25 \%-50 \%$ fibroid shrinkage, and greater than $90 \%$ uterine bleeding control in initial studies based in Europe (PEARL trial). ${ }^{18,24}$ In Europe and Canada, this is the only SPRM that has been approved and commercialized as a 3-month pre-operative adjuvant therapy and, more recently, as intermittent treatment of moderate to severe fibroid symptoms for up to four courses. ${ }^{24}$ However, it is not currently available in other countries such as the United States, in part because of concerns over rare but severe cases of liver toxicity. ${ }^{24}$ As anovulation was seen in $80 \%$ of women at the current doses of ulipristal acetate, the use of a reliable form of birth control is recommended. ${ }^{24}$ Some of the contraindications to this medication are severe asthma and liver impairment. Other SPRM members such as mifepristone, asoprisnil, vilaprisan and telapristone acetate have been investigated in phase 2 clinical trials with promising preliminary results. ${ }^{18,25} \mathrm{~A}$ main concern for all progesterone-inhibiting agents is the potential for induced endometrial growth by the unopposed estrogens, which could lead to the development of endometrial hyperplasia/cancer, although not confirmed by clinical trials, and other non-malignant reversible histological findings. ${ }^{24}$ For this reason, their long-term treatment safety profile is still under investigation.

\subsection{6 | GnRH agonists and antagonists}

Among the $\mathrm{GnRH}$ agonists, leuprolide acetate is the most commonly used as a $3.75 \mathrm{mg}$ monthly or $11.25 \mathrm{mg}$ every 3 months intramuscular injection for 3-6 months for the pre-operative treatment of uterine fibroids. ${ }^{26}$

$\mathrm{GnRH}$ agonists, after an initial stimulation of gonadotropin release ("flare effect"), induce a pituitary downregulation which decreases the production of gonadotropins and gonadal steroids, and inhibits further fibroid growth. GnRH agonists lead to amenorrhea in most women (>98\%) and are associated with a 35\%-65\% decrease in fibroid size within 3 months of treatment initiation. In some cases, this may allow for a minimally invasive surgical approach and may increase preoperative hemoglobin levels. ${ }^{17,26}$ However, by medically inducing menopause, GnRH agonists without add-back therapy are associated with adverse effects including hot flushes, mood swings, vaginal dryness, decreased libido, sleep disturbances, and bone loss in the case of long-term use (>6 months). ${ }^{26}$ To alleviate some of these adverse effects, hormonal add-back therapy can be used with good success. ${ }^{26}$

At the present time, the use of $\mathrm{GnRH}$ antagonists such as cetrorelix and ganirelix acetate is uncommon. ${ }^{26}$ Despite their immediate clinical response, they are more expensive than $\mathrm{GnRH}$ agonists, and have a shorter half-life requiring daily injections. ${ }^{26}$ However, a new orally administered $\mathrm{GnRH}$ antagonist (elagolix), which was approved in the United States as a drug for the treatment of moderate to severe endometriosis-associated pain, has been shown to be also effective in reducing menstrual bleeding and uterine volume in women with fibroids in a recently published clinical trial. ${ }^{27}$

\subsection{7 | Iron supplementation}

Since fibroids are often associated with heavy and prolonged menstrual bleeding, they can cause iron-deficiency anemia for which iron supplementation is a justified adjuvant treatment. ${ }^{13}$ If the diagnosis is confirmed, iron should be promptly initiated to reduce symptoms. ${ }^{28}$ Several oral formulations (150-200 mg of elemental iron daily) are available containing iron dextran, iron sucrose, or ferric gluconate. ${ }^{28}$ In severe or unresponsive cases, or in cases where the patient is unable to tolerate oral iron, intravenous iron can be an option although significantly more expensive and potentially unavailable in low-resource settings. ${ }^{28}$ The most common adverse effects of oral iron are gastrointestinal symptoms, while intravenous administration can cause allergic reaction (urticaria and pruritus) and musculoskeletal pain. Blood transfusion may be used as a last resort in unstable patients or after failed iron treatment. ${ }^{28}$

\subsection{8 | Aromatase inhibitors}

A promising class of agents for the treatment of uterine fibroids in pre-menopausal women includes aromatase inhibitors such as letrozole and anastrozole. However, at this time, there is limited evidence to support their broad and long-term use for fibroids. Aromatase inhibitors such as letrozole ( 2.5 or $5 \mathrm{mg}$ orally once a day) and 
anastrozole (10 mg orally once a day) induce a hypoestrogenic state by inhibiting the aromatization of androgens to estrogens which results in thinning of the endometrial lining and reduced menstrual bleeding. ${ }^{29}$ A Cochrane review on aromatase inhibitors efficacy has shown an approximate $40 \%-50 \%$ reduction in fibroid size, as well as an improvement in dysmenorrhea, menorrhagia, and duration of menses. ${ }^{29}$ Considering that the aromatase enzyme is expressed at higher concentrations in leiomyoma tissues from women of African ancestry, this therapy could potentially be even more effective in these populations. ${ }^{30}$ This class of agents is generally better tolerated than $\mathrm{GnRH}$ agonists, with a lower incidence of serious short-term anti-estrogenic adverse effects. ${ }^{29}$ However, at this time, there is insufficient evidence to support their broad and long-term use.

\section{2 | Interventional radiology procedures}

UAE and MRgFUS are effective minimally invasive treatments for fibroids in women who decline surgery or are not good surgical candidates. These outpatient or short hospital stay procedures have the benefit of quicker recovery time and fewer complications as compared with surgery.

\subsection{1 | Uterine artery embolization}

Uterine artery embolization (UAE) is a minimally invasive angiographic technique which uses tris-acryl gelatin microspheres or non-spherical polyvinyl alcohol to interrupt uterine blood supply which causes ischemic necrosis of the fibroids. ${ }^{31}$ Under light sedation, a small catheter is introduced via the right common femoral artery to reach and embolize the uterine arteries bilaterally. ${ }^{32}$ This procedure treats the whole uterus, rather than the fibroids specifically, and has the potential of permanently impairing uterine and ovarian function. ${ }^{33}$ Current pregnancy, suspected cancer, and active pelvic infections are absolute contraindications to this procedure. Women undergoing UAE reported an approximate $42 \%$ fibroid shrinkage at 3 months, shorter menstrual duration and improvement of bulk symptoms. ${ }^{17}$ Compared to myomectomy or hysterectomy, UAE is a shorter procedure with faster recovery and quicker return to normal activities. ${ }^{17}$ However, it is associated with a higher rate of minor complications (5\%), including ischemic injuries and fibroid expulsion, among others. ${ }^{32}$ Recurrence of fibroids at 5 years was also seen more frequently after UAE (20\%) rather than myomectomies, with more women requiring further interventions. ${ }^{32}$ One peculiar complication of UAE is the post-embolization syndrome caused by the release of ischemic fibroid products in the blood stream which can result in severe pain and fever and requires intense analgesic treatment and hydration. ${ }^{32}$

\subsection{2 | Magnetic resonance guided focused radiofrequency ablation}

MRgFUS, also known as high intensity focused ultrasound (HIFU), is an available but not largely adopted fibroid-specific therapy that uses high intensity transabdominal ultrasound waves to induce fibroid coagulative necrosis and regression. ${ }^{33}$ Because this procedure is performed under dynamic real-time MRI guidance, patients with pacemakers or other contraindications to MR scans are not eligible. Additionally, presence of five or more fibroids, pedunculated, gadolinium non-enhanced or very large fibroids $(>10 \mathrm{~cm})$, post-menopausal status, and severe adenomyosis are contraindications to this procedure. Following MRgFUS, approximately $71 \%$ of women reported an improvement of symptoms at 6 months. ${ }^{17,34}$ Although rare, reversible pelvic neuropathy and local skin burns are the main risks associated with MRgFUS. For the latter reason, the procedure is not recommended for women with prior extensive abdominal/pelvic surgeries or abdominal scarring. The rates of re-intervention following MRgFUS are higher (30.5\%) compared with myomectomies or UAE. ${ }^{34}$ Data on successful pregnancies after MRgFUS are rare and need further investigation. ${ }^{34}$

\section{3 | Surgical management}

Surgical management options include myomectomies, hysterectomies, laparoscopic radiofrequency volumetric thermal ablation, and endometrial ablation.

\subsection{1 | Myomectomy}

Myomectomy is a uterine-sparing procedure that involves removing the fibroids but leaving the uterus intact. It is most often offered to patients who desire future fertility but is also considered by those who have completed childbearing and wish to retain their uterus. Myomectomy provides temporary reduction in uterine volume and improvement of symptoms in up to $80 \%$ of women but is associated with an approximately $27 \%$ risk of recurrence after removal of a single fibroid, and greater than $50 \%$ in the case of multiple fibroids. ${ }^{35}$ Myomectomies are overall associated with a low rate of complications (1\%-5\%). ${ }^{36}$ The most common complication is high intraprocedural blood loss, for which intra-fibroid infiltration of vasopressin, intravaginal misoprostol or dinoprostone, the use of pro-fibrin/ thrombin agents, or the use of tourniquet around the cervix or infundibulo-pelvic ligaments have been demonstrated to decrease blood loss. ${ }^{36}$ Different approaches such as hysteroscopic, laparoscopic (including robotically assisted), and laparotomy can be undertaken to remove the fibroids. Surgical plans are made according to fibroid size, number, and location. Hysteroscopic myomectomies are the procedure of choice for the removal of smaller type 0 or type 1 submucosal fibroids. ${ }^{36}$ Type, size, and number of fibroids have been shown to predict the likelihood of achieving complete surgical resection and recurrence. ${ }^{17}$ Laparoscopic myomectomy, when feasible, should be the standard procedure for intramural and subserosal fibroids. This approach is associated with lower blood loss and morbidity, shorter hospital stay, and less post-operative pain than open myomectomies. ${ }^{37}$ For women who desire future childbearing and have significant uterine wall disruption during surgery, cesarean delivery prior to the onset of labor is recommended over vaginal delivery because of its association with an increased risk of 
a devastating maternal and neonatal consequence such as uterine rupture (approximate risk $<2 \%$ ). ${ }^{36}$

\subsection{2 | Hysterectomy}

Hysterectomy remains the only definitive surgical treatment for symptomatic fibroids. This is not an appropriate procedure for women who have not completed childbearing and/or who simply wish to retain their uterus. Following hysterectomy, the majority of women report a significant improvement in quality of life and symptomatology as early as 3 months after surgery. ${ }^{37}$ Vaginal, laparoscopic, robotic, and open approaches can be adopted. Vaginal and laparoscopic hysterectomies, when feasible, are recommended as first line because they are associated with shorter hospital stay, faster recovery and better patient satisfaction. ${ }^{37}$ During minimally invasive myomectomies and hysterectomies, sharp morcellation techniques can be used to remove fibroids and/or myomatous uteri in well counseled women. Although the prevalence of sarcoma is very rare in fibroids $(<0.3 \%)$, the risk of specimen fragment dispersion with morcellation remains a serious and highly debated concern that has been addressed by many international societies. ${ }^{18}$

Overall, hysterectomies are associated with a relatively low number of complications, including a $0.4 \%$ incidence of major complications, and low rate of reoperations for adhesions, pelvic prolapse or fistulas. $^{37}$

\subsection{3 | Laparoscopic radiofrequency volumetric thermal ablation}

Radiofrequency volumetric thermal ablation (RFVTA) is a laparoscopic outpatient procedure performed under ultrasound guidance which uses an electro-surgical probe, inserted within the fibroid, to induce coagulative myolysis. ${ }^{38}$ Available data demonstrate promising results indicating improvement in symptom severity and shrinkage of the fibroids (up to $77 \%$ volume reduction at 6 months) with a relatively low rate of reintervention (11\%), shorter hospital stay, and lower blood loss compared to laparoscopic myomectomies. ${ }^{38}$ However, there is still limited information regarding subsequent pregnancy or long-term outcomes and only few centers are currently offering this treatment option.

\subsection{4 | Endometrial ablation}

Good candidates for endometrial ablation, as an option for the management of fibroid-associated AUB, are pre-menopausal women who have completed childbearing. Thermal balloon, microwave, hydrothermablation, bipolar radiofrequency endometrial ablation, or endometrial cryotherapy devices are used to achieve endometrial destruction. ${ }^{39}$ Each device has specific criteria and recommendations for use mainly based on uterine cavity suitability. Desire for future fertility, active pelvic infections, endometrial cancer, and uterine congenital anomalies are contraindications for endometrial ablation. ${ }^{39}$ Since this procedure does not prevent future pregnancy and pregnancy after ablation can be associated with abnormal implantation/placentation and several obstetrical complications, a reliable method of contraception is recommended following this procedure. Endometrial ablation is associated with up to $90 \%$ satisfactory improvement of uterine bleeding, and low rate of complications (1\%-2\%) including uterine perforation and bleeding. ${ }^{39}$ Failure and reoperation rates range between $29 \%$ and $40 \%$ and are higher in cases of large intramural or submucosal fibroids, age older than 45 years, higher parity, history of dysmenorrhea or concomitant adenomyosis. ${ }^{39}$

\section{3 | FUTURE DIRECTIONS AND DISCUSSIONS}

Symptomatic uterine fibroids are associated with significant morbidity and can have a devastating impact on quality of life among women during their reproductive years. Because of their significant prevalence and associated health costs, fibroids constitute a growing public health issue worldwide. There are several medical, minimally invasive, and surgical treatment options for women with uterine fibroids that can help tailor the management of this condition to a patient's characteristics, symptoms, goals, and eligibility for therapy.

However, while some advances in research have broadened our knowledge on the pathophysiology of fibroids, additional studies are needed to better characterize their genotypes and phenotypes, develop new drug targets, perhaps compatible with fertility, and provide superior personalized care. Along these lines, future research should focus on investigating innovative pharmacological compounds targeting hormone receptors, the extracellular matrix, and novel molecules expressed by fibroids. More studies on comparative effectiveness, such as the COMPARE-UF study, ${ }^{40}$ are needed globally to optimize the right treatment for the right patient and to draw up evidence-based guidelines for the treatment of symptomatic fibroids in order to better guide physician counseling and patient therapy plans. Finally, further need exists for the determination of risk-stratification algorithms for women at high risk of developing fibroids in their lifetime, with the ultimate goal of not only treating, but also initiating primary preventive measures.

\section{AUTHOR CONTRIBUTIONS}

EG contributed to designing and writing the manuscript. SAS contributed to writing and revising the manuscript. EEM contributed to designing and editing the manuscript. All authors approved the final version and are accountable for all aspects of the work.

\section{ACKNOWLEDGMENTS}

EEM received funding from NIH (7R01MD011570) that helped support this work.

\section{CONFLICTS OF INTEREST}

EG has no conflicts of interest. SAS is a consultant for Abbvie, Myovant Sciences, and Bayer. EEM is a consultant for Myovant Sciences and has received funding from Allergan Dr. Marsh is currently receiving NIH 
funding through her grant 7R01MD011570 - ELLAS: Environment, Leiomyomas, Latinas and Adiposity.

\section{REFERENCES}

1. Bulun SE. Uterine fibroids. N Engl J Med. 2013;369:1344-1355.

2. Stewart EA. Clinical practice. Uterine fibroids. N Engl J Med. 2015;372:1646-1655.

3. Marsh EE, Bulun SE. Steroid hormones and leiomyomas. Obstet Gynecol Clin North Am. 2006;33:59-67.

4. Marsh EE, Ekpo GE, Cardozo ER, Brocks M, Dune T, Cohen LS. Racial differences in fibroid prevalence and ultrasound findings in asymptomatic young women (18-30 years old): A pilot study. Fertil Steril. 2013;99:1951-1957.

5. Stewart EA, Cookson CL, Gandolfo RA, Schulze-Rath R. Epidemiology of uterine fibroids: A systematic review. BJOG. 2017;124:15011512.

6. Baird DD, Dunson DB, Hill MC, Cousins D, Schectman JM. High cumulative incidence of uterine leiomyoma in black and white women: Ultrasound evidence. Am J Obstet Gynecol. 2003;188:100-107.

7. Cardozo ER, Clark AD, Banks NK, Henne MB, Stegmann BJ, Segars $\mathrm{JH}$. The estimated annual cost of uterine leiomyomata in the United States. Am J Obstet Gynecol. 2012;206:211.

8. Soliman AM, Yang H, Du EX, Kelkar SS, Winkel C. The direct and indirect costs of uterine fibroid tumors: A systematic review of the literature between 2000 and 2013. Am J Obstet Gynecol. 2015;213:141-160.

9. Marsh EE, Brocks ME, Ghant MS, Recht HS, Simon M. Prevalence and knowledge of heavy menstrual bleeding among African American women. Int J Gynecol Obstet. 2014;125:56-59.

10. Shen $\mathrm{Y}, \mathrm{Xu} \mathrm{Q}, \mathrm{Xu} J$, Ren ML, Cai YL. Environmental exposure and risk of uterine leiomyoma: An epidemiologic survey. Eur Rev Med Pharmacol Sci. 2013;17:3249-3256.

11. Segars JH, Parrott EC, Nagel JD, et al. Proceedings from the third national institutes of health international congress on advances in uterine leiomyoma research: Comprehensive review, conference summary and future recommendations. Hum Reprod Update. 2014;20:309-333.

12. Moravek MB, Yin P, Ono M, et al. Ovarian steroids, stem cells and uterine leiomyoma: Therapeutic implications. Hum Reprod Update. 2014;21:1-12.

13. Laughlin-Tommaso SK, Stewart EA. Moving toward individualized medicine for uterine leiomyomas. Obstet Gynecol. 2018;132: 961-971.

14. Levens ED, Wesley R, Premkumar A, Blocker W, Nieman LK. Magnetic resonance imaging and transvaginal ultrasound for determining fibroid burden: Implications for research and clinical care. Am J Obstet Gynecol. 2009;200:537.

15. Ghant MS, Sengoba KS, Recht H, Cameron KA, Lawson AK, Marsh EE. Beyond the physical: A qualitative assessment of the burden of symptomatic uterine fibroids on women's emotional and psychosocial health. J Psychosom Res. 2015;78:499-503.

16. Munro MG, Critchley HOD, Broder MS, Fraser IS. FIGO classification system (PALM-COEIN) for causes of abnormal uterine bleeding in nongravid women of reproductive age; for the FIGO working group on menstrual disorders. Int J Gynecol Obstet. 2011;113:3-13.

17. American College of Obstetricians and Gynecologists. ACOG practice bulletin. Alternatives to hysterectomy in the management of leiomyomas. Obstet Gynecol. 2008;112(2 Pt 1):387-400.

18. Donnez J, Dolmans M-M. Uterine fibroids management: From the present to the future. Hum Reprod Update. 2016;22:665-686.

19. Sohn GS, Cho S, Kim YM, et al. Current medical treatment of uterine fibroids. Obstet Gynecol Sci. 2018;61:192-201.
20. Lethaby A, Duckitt K, Farquhar C. Non-steroidal anti-inflammatory drugs for heavy menstrual bleeding. Cochrane Database Syst Rev. 2013;(1):CD000400.

21. Lukes AS, Moore KA, Muse KN, et al. Tranexamic acid treatment for heavy menstrual bleeding: A randomized controlled trial. Obstet Gynecol. 2010;116:865-875.

22. Moroni RM, Martins WP, Dias SV, et al. Combined oral contraceptive for treatment of women with uterine fibroids and abnormal uterine bleeding: A systematic review. Gynecol Obstet Invest. 2015;79:145-152.

23. Jiang $\mathrm{W}$, Shen $\mathrm{Q}$, Chen $\mathrm{M}$, et al. Levonorgestrel-releasing intrauterine system use in premenopausal women with symptomatic uterine leiomyoma: A systematic review. Steroids. 2014;86:69-78.

24. Donnez J, Tatarchuk TF, Bouchard P, et al. Ulipristal acetate versus placebo for fibroid treatment before surgery. $N$ Engl J Med. 2012;366:409-420.

25. Murji A, Whitaker L, Chow TL, Sobel ML. Selective progesterone receptor modulators (SPRMs) for uterine fibroids. Cochrane Database Syst Rev. 2017;(4):CD010770.

26. Lethaby A, Vollenhoven B, Sowter M. Pre-operative GnRH analogue therapy before hysterectomy or myomectomy for uterine fibroids. Cochrane Database Syst Rev. 2001;(2):CD000547.

27. Archer DF, Stewart EA, Jain RI, et al. Elagolix for the management of heavy menstrual bleeding associated with uterine fibroids: Results from a phase 2a proof-of-concept study. Fertil Steril. 2017;108:152-160.

28. Low MSY, Speedy J, Styles CE, De-Regil LM, Pasricha SR. Daily iron supplementation for improving anaemia, iron status and health in menstruating women. Cochrane Database Syst Rev. 2016;(4):CD009747.

29. Song H, Lu D, Navaratnam K, Shi G. Aromatase inhibitors for uterine fibroids. Cochrane Database Syst Rev. 2013;(10):CD009505.

30. Ishikawa H, Reierstad S, Demura M, et al. High aromatase expression in uterine leiomyoma tissues of African American women. J Clin Endocrinol Metab. 2009;94:1752-1756.

31. Gupta JK, Sinha A, Lumsden MA, Hickey M. Uterine artery embolization for symptomatic uterine fibroids. Cochrane Database Syst Rev. 2014;(12):CD005073.

32. De Bruijn AM, Ankum WM, Reekers JA, et al. Uterine artery embolization vs. hysterectomy in the treatment of symptomatic uterine fibroids: 10-year outcomes from the randomized EMMY trial. Am J Obstet Gynecol. 2016;215:745.e1-745.e12.

33. Lee BB, Yu SP. Radiofrequency ablation of uterine fibroids: A review. Curr Obstet Gynecol Rep. 2016;5:318-324.

34. Sandberg EM, Tummers FHMP, Cohen SL, van den Haak L, Dekkers $\mathrm{OM}$, Jansen FW. Reintervention risk and quality of life outcomes after uterine-sparing interventions for fibroids: A systematic review and meta-analysis. Fertil Steril. 2018;109:698-707.

35. Kotani Y, Tobiume T, Fujishima R, et al. Recurrence of uterine myoma after myomectomy: Open myomectomy versus laparoscopic myomectomy. J Obstet Gynaecol Res. 2018;44:298-302.

36. Bhave Chittawar P, Franik S, Pouwer AW, Farquhar C. Minimally invasive surgical techniques versus open myomectomy for uterine fibroids. Cochrane Database Syst Rev. 2014;(10):CD004638.

37. Pitter MC, Simmonds C, Seshadri-Kreaden U, Hubert HB. The impact of different surgical modalities for hysterectomy on satisfaction and patient reported outcomes. J Med Internet Res. 2014;3:e11.

38. Havryliuk Y, Setton R, Carlow JJ, Shaktman BD. Symptomatic fibroid management: Systematic review of the literature. JSLS. 2017;21:e2017. 00041.

39. Munro MG. Endometrial ablation. Best Pract Res Clin Obstet Gynaecol. 2018;46:120-139.

40. Stewart EA, Lytle BL, Thomas L, et al. The comparing options for management: patient-centered results for uterine fibroids (COMPARE-UF) registry: rationale and design. Am J Obstet Gynecol. 2018;219:95. e1-95.e10. 